\title{
MEMPELAJARI STRUKTUR DAN SIFAT NANO LOGAM TEMBAGA MENGGUNAKAN PROGRAM PM3
}

\author{
Mulyati Sukma, Theresia Sita Kusuma dan Syukri Arief \\ Laboratorium Komputasi Jurusan Kimia FMIPA Unand \\ Kampus Unand Limau Manis Padang Sumbar
}

\begin{abstract}
A research to study the electronic and chemical characteristics of Copper which nano structure using PM3 program has been done. This program part of HyperChem pro 6.0 that performed by Pentium 4. This research has nano 2D and 3D structure (atom number 2-30). Results of computations showed that Copper can make nanosheets, nanorods and nanotubes structure that have the different electronic characteristics from its crystals structure. Fortunately, copper with nano structure could develop as insulator and semiconductor materials.
\end{abstract}

Keywords : Copper, nano structure, nanosheets, nanorods, nanotubes. 


\section{DAFTAR PUSTAKA}

1. Wei-Na, L., Yuan, J., Mower, S. G., Sithambaran, S., and Suib, S. L., 2006, $J$. Pys. Chem. B., 110: 3066.

2. Darugar, Q., Qian, W., El-Sayed, M. A., 2006, J. Phys. Chem.B.,110: 143.

3. Miyawaki, J., Ydasaka, M., Imai, H., Yorimitzu, H., Isobe, H., Nakamura, E., and Iijima, S., 2006, J. Phys. Chem. B.,110: 5179 .

4. Zhou, Z., Zhao, J., Chen, Z., Gao, X, Lu, J. P, Schleyer, P. R., and Yang, C-K., 2006, J. Phys. Chem.B., 110: 2529.
5. Chen, X., Gao, P., Zhang, H., Zhou, Z., Hu, W. K., Pan, G. L., Zhu, H. Y., Yan, T. Y., and Song, D. Y., 2005, J. Phys. Chem. B., 109: 11525-11529.

6. Pawluk, T., Hirata, J., Wang, L., 2005, J. Phys. Chem. B., 109: 20817.

7. Xiao, L., and Wang, L., 2004, J. Phys. Chem. A., 108: 8605.

8. Kusuma, S. T., 2006, Unpublished Results.

9. Kittle, C., 1971. Introduction To Solid State Physics, $4^{\text {th }}$ ed. Willey, New York.

10. HyperChem Release 6,0 for Windows, 1996, Hypercube Inc. 